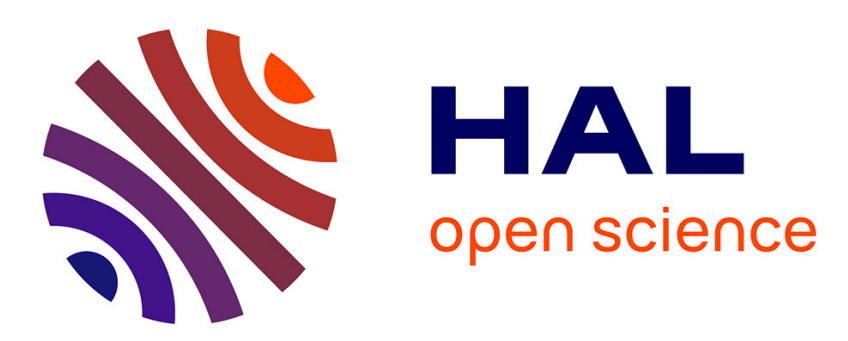

\title{
Modelling of the rheological behavior of mechanically dewatered sewage sludge in uniaxial cyclic compression
}

Fenglin Liang, Martial Sauceau, Gilles Dusserre, Jean-Louis Dirion, Patricia Arlabosse

\section{- To cite this version:}

Fenglin Liang, Martial Sauceau, Gilles Dusserre, Jean-Louis Dirion, Patricia Arlabosse. Modelling of the rheological behavior of mechanically dewatered sewage sludge in uniaxial cyclic compression. Water Research, 2018, 147, p.413-421. 10.1016/j.watres.2018.10.016 . hal-01898128

\section{HAL Id: hal-01898128 https://imt-mines-albi.hal.science/hal-01898128}

Submitted on 7 Nov 2018

HAL is a multi-disciplinary open access archive for the deposit and dissemination of scientific research documents, whether they are published or not. The documents may come from teaching and research institutions in France or abroad, or from public or private research centers.
L'archive ouverte pluridisciplinaire HAL, est destinée au dépôt et à la diffusion de documents scientifiques de niveau recherche, publiés ou non, émanant des établissements d'enseignement et de recherche français ou étrangers, des laboratoires publics ou privés. 


\title{
Modelling of the rheological behavior of mechanically dewatered sewage sludge in uniaxial cyclic compression
}

\author{
Fenglin Liang a, Martial Sauceau ${ }^{\text {a, *, Gilles Dusserre }}{ }^{\text {b }}$, Jean-Louis Dirion ${ }^{\text {a }}$, \\ Patricia Arlabosse ${ }^{a}$ \\ ${ }^{a}$ Université de Toulouse, Mines Albi, CNRS, Centre RAPSODEE, Campus Jarlard, 81013, Albi, France \\ ${ }^{\mathrm{b}}$ Institut Clément Ader (ICA), Université de Toulouse, CNRS, Mines Albi, UPS, INSA ISAE-SUPAERO, Campus Jarlard, 81013, Albi CT, Cedex 09, France
}

\begin{abstract}
A B S T R A C T
The rheological behavior of mechanically dewatered sewage sludges is complex but essential as it affects almost all treatment, utilization and disposal operations, such as storage, pumping, land-spreading, or drying. In this work, a specific methodology coupling experiments and modelling is developed to characterize the rheological and textural properties of highly concentrated sludge. The experimental part based on a uniaxial compression method has been presented in a previous paper (Liang et al., 2017). This article is dedicated to the modelling part, which includes the behavior identification and the parameters optimization. Previous and additional mechanical tests allow the identification of a visco-elasto-plastic behavior. This behavior is then modelled with a Burgers-Ludwik model, with 7 rheological parameters. This model is able to simulate the viscoelastic behavior of sludge under the yield stress, and the visco-elasto-plastic hardening behavior over the yield stress. The optimization of model parameters is carried out in two steps and relies on the calculation of basins of attraction and confidence intervals with initial conditions estimated from the mechanical tests. Finally, the entire characterization methodology, from experimental mechanical tests to model parameter optimization, is applied to sludge samples at different operating conditions and structural states. The determination of the rheological properties of sludge is achieved with excellent matching between simulation and experimental results. Being able to take into account these impact factors, the rheological model can be used to predict the sludge behavior in various operating conditions.
\end{abstract}

Keywords:

Uniaxial compression

Rheological modelling

Visco-elasto-plastic behavior

Burgers model

Ludwik equation

\section{Introduction}

In France, with the progressive implementation of the municipal wastewater treatment Directive 91/27/EEC, an excessive quantity of sewage sludge is produced, about 1 million tons of dry matter in 2014 (Eurostat), necessitating further disposal. These solids and biosolids originating from the processes of treatment contain only $0.25-5 \%$ of solid matter by weight. In order to reduce its volume, namely the cost for handling, transport and storage, thickening, conditioning and mechanical dewatering are necessary treatment to decrease moisture content in the first place. The sewage sludge in form of a liquid becomes more and more viscous with increasing total solid content (TS), resulting in the decrease of flowability. When the TS reaches $18-25 \%$ after these dewatering processes, the sludge exceeding its liquid limit can no longer flow under the effect

\footnotetext{
* Corresponding author.

E-mail address: Martial.Sauceau@mines-albi.fr (M. Sauceau).
}

of gravity, but maintains its shape as a solid (Liang et al., 2017). Only if submitted to a high enough stress, it could continuously deform as yield stress fluids. The primary difference between yield stress fluids and dewatered sewage sludge is that the latter possesses significant plastic behavior: it cannot completely recover its initial properties after strain relief (Coussot, 2014). As the solidlike sludge is highly resistant to flow, it makes the flow control a real challenge in pumping, land spreading and drying (Baudez et al., 1998; Lotito and Lotito, 2014). Furthermore, the aging of organic matter inside sludge may also modify its rheological and textural properties (Liang et al., 2017). Consequently, the quantitative characterization of rheological properties (e.g. elastic module, viscosity, yield stress, etc.) of mechanically dewatered sludge over liquid limit, in various operating and storage conditions, became the determinant issue in processing engineering and optimization.

However, the conventional rheometry by shearing is appropriate to measure homogeneous material in liquid or fluid state 
with TS lower than 16\% (Chhabra and Richardon, 2011; Tadros, 2010; Malkin and Isayev, 2006; Morrison, 2001; Tanner and Walters, 1998; Coussot, 1997). Moreover, different protocols of rheometrical measurement may lead to different results. For instance, Jiang et al. (2014) tested with parallel plates geometry 4 sludge samples of TS about $8,10,13$ and $16 \%$ with both flow and dynamic methods and the yield stresses obtained in dynamic measurement were $35,12,75$ and $32 \%$ higher, respectively: whereas, Mori et al. (2006) observed that the yield stresses of several samples of TS in the range $2.7-5.7 \%$ are from 13 to $50 \%$ lower in dynamic measurement with concentric cylinders geometry. In another study, the same authors detailed the applicability of concentric cylinders, double concentric cylinders and helical ribbon impeller systems in characterizing sludge of TS in the range 0.4-4.3\% (Mori et al., 2008). Ratkovich et al. (2013) also reviewed a vast amount of papers to highlight the impacts of rheometer choice, rheometer settings and measurement protocol on varying conclusions. Inevitably, this difficulty is then transferred to the modelling of the sludge behavior, which is always based on empirical equations for a qualitative analysis, such as the Herschel-Bulkley model initially used for yield stress fluids and the Cross model for polymers (Jiang et al., 2014; Mori et al., 2008; Eshtiaghi et al., 2013; Seyssiecq et al., 2003). Aiming at these uncertainties, it is extremely pre-requisite to develop an appropriative methodology from reproducible experimental protocol, rigorous data analysis to theoretical modelling with fundamental physical laws to determine the rheological properties of solidlike sewage sludge.

In a previous paper, a uniaxial compression method was developed to characterize the rheological behaviors of sewage sludge with TS between the liquid and plastic limits (Liang et al., 2017). The protocols from sludge sampling to testing machine settings of the mechanical tests and the methods of sludge rheological behavior identification, based on the experimental true stress-true strain curves, were detailed, and the primary estimations of elastic modulus, viscosity and yield stress orders of magnitude of tested samples were illustrated and verified.

Analogical models based on fundamental mechanics are generally adopted to simulate material behavior under compression tests. They are constituted of three mechanical elements: the spring used to represent the Hooke's law for an ideal elastic body; the dashpot to stand for the Newton's law defining the viscosity; and the slider to describe the plastic behavior with yielding point. The numerous possible combinations of these elements allow representing a large variety of materials used in several application fields. Lin et al. (2013) used the generalized Maxwell model to simulate the viscoelastic relaxation behavior of sludge granules under compression tests. More recently, the viscoelastic behavior of mechanically dewatered high-solid sludge (TS of 14.2 and $18.2 \%$ ) was well modelled by the Kaye-Bernstein-Kearsly-Zapas (KBKZ) model with paralleled Maxwell elements. This model was used to describe the frequency dependence of elastic modulus and viscous modulus during shear creep tests (Zhang et al., 2017). In rheology literature, it is also customary to consider the so-called Burgers model, which consists of a Maxwell and a Kelvin materials in series (Malkin and Isayev, 2006; Morrison, 2001; Barnes et al., 1998). Indeed, this model is able to represent the rheological behavior of various materials as altered rock (Jiang et al., 2015), epoxy adhesive (Costa and Banos, 2015), asphalt mixture (Cai et al., 2013), thermoplastic polyurethane/organoclay nanocomposite (Ercan et al., 2017) or flour doughs (Moreira et al., 2015; Meerts et al., 2017). However, the Burgers model can require additional elements to represent a particular characteristic of the rheological behavior, as for instance an empirical expression for the stress hardening behavior of agar gels (Yu et al., 2012) or a plastic element for sandstone samples (Zhang et al., 2014). The main limitation of such uniaxial models is that they are unable to account for the material behavior under any triaxial state of stress. However a thorough characterization of the main features of the behavior under uniaxial load is the first step toward a unified model able to account for combined shear and compression.

By reference to these studies, in the present work, complementary creep and relaxation tests are firstly adapted to identify the sludge rheological properties in a longer scale of time (over $30 \mathrm{~min}$, duration typically encountered in practical handling) with respect to the cyclic compression tests (less than $30 \mathrm{~s}$ ). These tests can also reveal the creep and relaxation behaviors of sludge samples that are essential in establishing the analogical model. Then, the identified visco-elasto-plastic behaviors and the estimated rheological properties derived from cyclic compression tests are exploited to build up a reliable model so as to simulate sludge behaviors. In particular, a specific model parameter optimization procedure is developed based on calculating the basins of attraction to determine the exact values of rheological properties of tested samples. Finally, the entire characterization methodology is applied to determine the rheological properties of sludge samples at various operating conditions and to predict the solidlike sludge performance in various operating conditions.

\section{Material and method}

\subsection{Origin of mechanically dewatered sewage sludge and sample preparation}

The mechanically dewatered sewage sludge was sampled at the wastewater treatment plant (WWTP) of Albi (France). It is produced from extendedly aerated, thickened and digested municipal wastewater (Liang et al., 2017). The sludge was collected at the outlet of the centrifuge and then transported immediately to the laboratory within $1 \mathrm{~h}$ for testing. Its initial total solid (TS, standard EN 12880:2000) content was ranged from 18.5 to $21 \mathrm{wt} \%$ and the volatile solid (VS, standard EN 12879:2000) content is of about $63 \%$ (of dry weight). It was shaped into cylinders using a manual extruder according to a procedure previously described (Liang et al., 2017). Finally, all the extruded sludge samples have a density of $1060 \mathrm{~kg} / \mathrm{m}^{3}$ and the same dimensions, with a radius $\mathrm{r}_{0}=17 \mathrm{~mm}$ and a height $\mathrm{h}_{0}=51 \mathrm{~mm}$, corresponding to the aspect ratio $h_{0} / 2 r_{0}=1.5$. All the experiments were carried out in the airconditioned laboratory at $21^{\circ} \mathrm{C}$.

Three identical cylindrical samples were measured for each test (creep and relaxation). The curve in the middle of the three resulting curves was used for behavior analysis. The TS and the temperature of sludge samples were surveyed from sample preparation to the end of mechanical tests. Because of moisture evaporation, the TS increased from 18.9 to $19.4 \%$ and the temperature of the samples decreased from 20.4 to about $18^{\circ} \mathrm{C}$ after 90 min' tests. To avoid significant impact due to water evaporation on samples' behaviors, the duration of tests was limited to $30 \mathrm{~min}$. As in the cyclic compression tests (Liang et al., 2017), all the tested samples were also examined visually to check the absence of external and internal fractures by cutting them into slices, and to guarantee that there was no significant impact on sludge behavior analysis.

\subsection{Creep test by uniaxial compression}

The creep test measures the evolution of strain in function of time under a constant applied stress. It reveals the effects of material viscosity in short, mid and long time under stable loading condition.

The universal testing machine (UTM) LRX Plus of Lloyd Instrument was used to carry out the creep tests. The same material- 
testing machine was used for the previous uniaxial cyclic compression tests (Liang et al., 2017). As the machine can only impose the force $\mathrm{F}(\mathrm{N})$ and the deformation $\Delta h(\mathrm{~mm})$, the constant loading force was controlled at low values ( 0.5 and $1 \mathrm{~N})$ which may not generate large deformations on sludge samples. In consequence, the applied stress could be considered as nearly constant.

\subsection{Relaxation test by uniaxial compression}

The relaxation test measures the evolution of stress in function of time at a constant strain. It reveals the coupled effects of material viscosity and elasticity.

This test was also performed with the UTM by pre-setting the loading force at 1 and $2 \mathrm{~N}$. The maximum strains generated by the two forces were then maintained unchanged to obtain the relaxation conditions.

\subsection{Sludge rheological behavior modelling}

Rheological models are built up from three analogical mechanic elements, Fig. 1: spring, dashpot and slider, representing respectively a perfect elastic body, an ideal viscous one and a plastic one with a yield stress.

As it concerns here uniaxial compression tests, the constitutive equation between the instantaneous stress, $\sigma(t)(\mathrm{Pa})$, and strain, $\varepsilon(t)(-)$, of each element will be written in one-dimension.

The spring complies with the Hooke's law, Equation (1), where E (Pa) is the elastic modulus:

$\sigma(t)=E \cdot \varepsilon(t)$

A linear dashpot is also called a Newtonian dashpot. Its constitutive equation is set up with the Newton's law, Equation (2). $\eta$ (Pa.s) is the viscosity:

$\sigma(t)=\eta \frac{d \varepsilon(t)}{d t}$

The slider can simulate the yielding behavior (yield stress $\sigma_{c}$ (Pa)) of a plastic body: when the applied stress is smaller than the yield stress, the slider stays blocked; while higher than the yield stress, the slider starts to displace, the stress $\sigma(t)(\mathrm{Pa})$ becomes a function of the plastic strain $\varepsilon_{p}(t)(-)$, Equation (3).

$|\sigma|=\sigma_{c}=f\left(\varepsilon_{p}\right), \dot{\varepsilon}_{p}>0$

After the experimental characterization of the sludge, a rheological model will be developed to be able to represent the observed behavior of the sludge. This model will be composed of an association in series and/or parallel of analogical mechanic elements. The constitutive equations of the model can be then expressed by using the following rules: when these elements are connected in series, the stress in each component is identical and the strains are cumulative, while in parallel, the stresses are cumulative and the strain is identical. The stress being identical in each block of the model, the measured stress data $\sigma_{\exp }$ are considered as given conditions and the model is implemented to calculate the strain $\varepsilon_{\text {cal }}$.

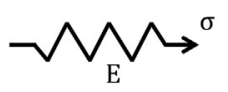

(a)

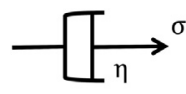

(b)

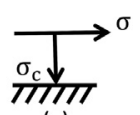

(c)
Fig. 1. Symbols of the three analogical mechanic elements: (a) spring; (b) dashpot; (c) slider.

\subsection{Optimization procedure of model parameters}

Once the elements of the model are identified, the values of the $\mathrm{m}$ parameters have to be determined. The nonlinear data-fitting is programmed with "Isqnonlin" function of the software MATLAB ${ }^{\circledR}$ which uses the least-squares method. For each experiment, the strain $\varepsilon_{\text {cal }}$ is calculated by using the constitutive equations of the model and preset values of the model parameters $(\mathrm{P})$ in order to minimize the residual (noted as Residu) defined as following:

Residu $\left.=\sum_{1} \frac{\varepsilon_{c a l}^{i}-\varepsilon_{\mathrm{exp}}^{i}}{\varepsilon_{\exp }^{i}}\right)^{2}$

It is equal to the squared difference between experimental results $\varepsilon_{\exp }$ and calculated values $\varepsilon_{\text {cal }}$. The program varies the values of model parameters $P$ until Residu or the difference between two consecutive values ( $\Delta$ Residu) is lower than $10^{-6}$.

The constitutive equation of the model is a differential equation of second order that may have more than one attractor, thus the variation on initial values of the model parameters may lead to different attractor. At first, the value of each parameter is estimated on the basis of the results obtained in uniaxial cyclic compression (Liang et al., 2017), creep and relaxation tests of this work. These estimated values $P_{i}$ are used as basic order of magnitude to define the testing ranges of the $\mathrm{m}$ model parameters. Then, the basins of attraction are calculated to verify the uniqueness of attractor in reasonable ranges of model parameters. These ranges are fixed to $\pm 50 \%$ of the estimated values $P_{i}$. The initial values of all the parameters are thus preset at 3 levels $P_{P}$ equal to $0.5 P_{i}, P_{i}$ and $1.5 P_{i}$, respectively. There are thus in total $\mathrm{n}=3^{\mathrm{m}}$ sets of initial values to be evaluated in multiple regression calculations with the same algorithm to obtain the same number of groups of identified model parameters. As each parameter is independently adjusted to $\pm 50 \%$ of its basic order of magnitude, this method can simultaneously evaluate the sensibility of model parameters. The sets of optimized model parameters $P$ are then used to calculate the confidence interval $C l_{\alpha}$ at the significance level $\alpha$ :

$C I_{\alpha}=\left[\bar{P}-T_{\frac{\alpha}{2}}^{n-m} S_{P} ; \bar{P}+T_{\frac{\alpha}{2}}^{n-m} S_{P}\right]$

$\bar{P}$ is the average of optimized parameters, $\mathrm{n}$ the number of sets of initial model parameters, $m$ the number of model parameters and $S_{P}$ the square root of the variance of $\mathrm{P}$, and $t_{\frac{\alpha}{2}}^{n-m}$ the fractile of order $1-\alpha / 2$ of the Student's t-distribution at $(n-m)$ degrees of freedom.

\section{Characterization and modelling of the rheological behavior}

\subsection{Creep and relaxation tests analysis}

In our previous study, two rheological behaviors and three types of deformation were identified (Liang et al., 2017) in the one cycle of loading-unloading uniaxial compression test:

- The viscoelastic behavior below $\sigma_{\mathrm{c}}$ in the loading phase and the entire unloading phase and the visco-elasto-plastic behavior above $\sigma_{\mathrm{c}}$ in the loading phase;

- The instantaneous and deferred recoverable deformations due to the viscoelastic property of sludge and the permanent deformation due to the plastic property.

This analysis aims to complete the sludge rheological behavior identified from the uniaxial cyclic compression test, because the cyclic compression test only lasts for a maximum of $30 \mathrm{~s}$. This 
responsive time is not long enough in comparison with the scale of residence time of sludge in practical handling. This is the reason for which the creep and relaxation tests are necessary for the rheological characterization and modelling of sludge.

The strain is plotted in function of time during the creep test (Fig. 2(a)). The sludge samples exhibit firstly the primary creeping behavior like a solid, since the slopes of the two strain-time curves decrease rapidly in the first $2 \mathrm{~min}$ (Liang et al., 2017). Then, the slope of the curve tends to a constant value, corresponding to the secondary creeping behavior like a fluid, also known as the linear creeping. The sludge exhibits thus the characteristics of both fluid and solid.

The Maxwell model, a spring and a dashpot in series, is the simplest model to simulate the linear creeping behavior. When the stress is fixed at $\sigma_{\mathrm{f} 0}$, the creeping strain $\varepsilon_{\mathrm{f}}(\mathrm{t})$ can be calculated with Equation (6):

$\varepsilon_{\mathbf{f}}(\mathbf{t})=\frac{\sigma_{\mathbf{f} 0}}{\eta_{3}} \mathbf{t}+\varepsilon_{\mathbf{f} 0}$

where $\varepsilon_{\mathrm{fo}}$ is the initial condition of dimensionless strain in the linear creep and $\eta_{3}$ is the viscosity of the dashpot (linear creeping on Fig. 2(a)). The slope of this linear creep seems to be independent of elastic property of material. The slope of this linear creeping curve is the ratio between the applied stress and the viscosity. Knowing the value of $\sigma_{\mathrm{f} 0}$ and the slope (by linear regression analysis), it can be deduced that $\eta_{3}$ is in the order of magnitude of $10^{7} \mathrm{~Pa} \mathrm{~s}$. This value is close to that of food gels equal to approximately $10^{6} \mathrm{Pas}$ (Yu et al., 2012), some sandstone at about $10^{6}-10^{9}$ Pa s (Zhang et al., 2014) and bitumen at about $10^{8} \mathrm{~Pa} \mathrm{~s}$ (Chhabra and Richardson, 2011). These materials are all very deformable ones with similar texture as our sludge. So the estimation of the sludge viscosity $\eta_{3}$ seems to be appropriate and reliable and it can be used as an initial value in model optimization.

In relaxation tests, the stress continues to decrease after $30 \mathrm{~min}$ relaxation (Fig. 2(b)). It tends neither to zero as a fluid, nor to a constant as a solid. Once again, the sludge is proved to be an intermediate material between fluid and solid.

The simplest relaxation can also be simulated with the Maxwell model. When the strain is fixed at $\varepsilon_{\mathrm{r} 0}$, the stress $\sigma_{\mathrm{r}}(\mathrm{t})$ can be calculated with Equation (7):

$\sigma_{\mathbf{r}}(\mathbf{t})=\sigma_{\mathbf{r} 0} \mathbf{e}^{-\mathbf{t} / \tau_{M}}$ with $\tau_{\mathbf{M}}=\frac{\eta_{3}}{\mathbf{E}_{1}}$

where $\sigma_{\mathrm{r} 0}$ is the initial stress of the relaxation (dependent of $\varepsilon_{\mathrm{r} 0}$ )

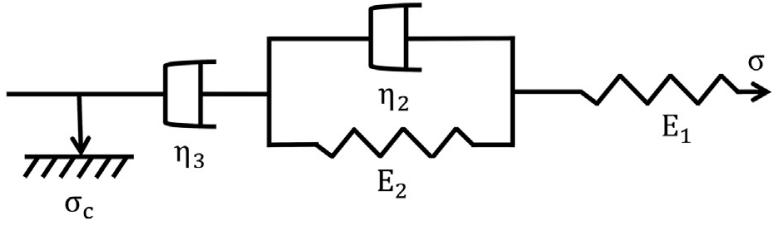

Fig. 3. Configuration of the Burgers-Ludwik model to simulate the visco-elasto-plastic behavior of sewage sludge in uniaxial cyclic compression.

and $\tau_{\mathrm{M}}$ is the characteristic relaxation time of the Maxwell model depending on the ratio between the dashpot viscosity and spring elasticity. From the experimental results, the stress decreases from 1070 to $400 \mathrm{~Pa}$ after $10 \mathrm{~min}$ of relaxation for the test at 0.03 . So it can be deduced (from Equation (7)) that the relaxation time is about $10^{2} \mathrm{~s}$. As the elastic modulus of $\mathrm{E}_{1}$ is in the order of magnitude of $10^{4}-10^{5} \mathrm{~Pa}$ (Liang et al., 2017), the viscosity $\eta_{3}$ is estimated at about $10^{6}-10^{7} \mathrm{~Pa} s$, a value similar to the previous estimation in creeping tests.

Finally, with these additional mechanical tests, the identification of sludge rheological properties is completed with consistent order of magnitude. A rheological model can be now developed to simulate the behavior of the sludge.

\subsection{Modelling conception of sludge behavior in uniaxial cyclic compression}

To fully reproduce the rheological behavior of sludge going through uniaxial cyclical compression, the modelling is carried out in two parts: the viscoelastic behavior in both loading (below $\sigma_{\mathrm{c}}$ ) and unloading phases, then the visco-elasto-plastic behavior in the loading (above $\sigma_{\mathrm{c}}$ ) phase.

\subsubsection{Viscoelastic behavior modelling}

The model is represented on Fig. 3. The instantaneous recoverable deformation behavior can be directly simulated with a spring $\mathrm{E}_{1}$. The deferred recoverable behavior can be modelled by KelvinVoigt composed of the connection in parallel of a spring $E_{2}$ and a dashpot $\eta_{2}$, the dashpot $\eta_{2}$ delaying the deformation of the spring $\mathrm{E}_{2}$. As previously specified, this model can predict the primary creep of a viscoelastic solid. Moreover, its deformation is entirely recoverable with the characteristic relaxation time $\eta_{2} / E_{2}$.

In order to reproduce the complex creep and relaxation characteristics of the viscoelastic property of sludge (section 3.1), a

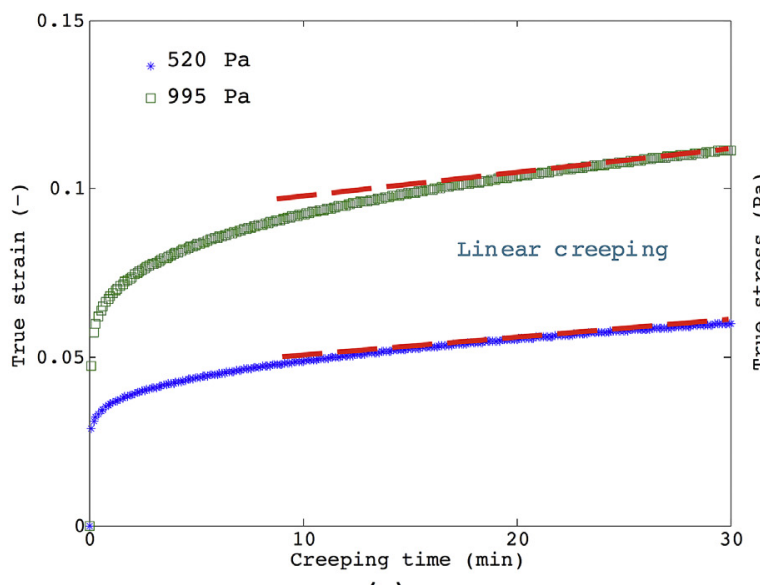

(a)

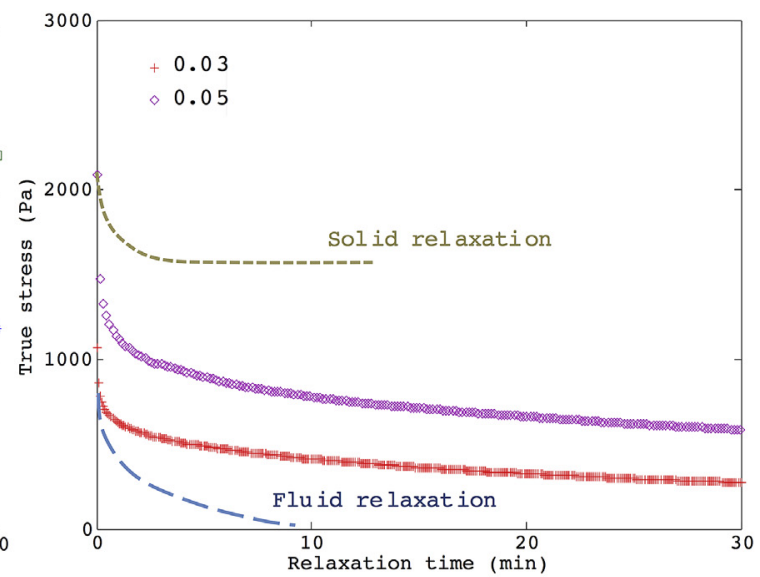

(b)

Fig. 2. (a) Creeping behavior of sludge at constant stresses 520 and $995 \mathrm{~Pa}$; (b) relaxation behavior of sludge at constant strains 0.03 and 0.05 . 
dashpot $\eta_{3}$ is added to the previous components. The connection in series of the spring $E_{1}$ and the dashpot $\eta_{3}$ forms the so-called Maxwell model, which can simulate at the same time a secondary (linear) creep behavior and an exponential relaxation of stress.

On the whole, the assembly of the four elements $E_{1}, E_{2}, \eta_{2}$ and $\eta_{3}$ is actually known as the Burgers model. It has two characteristic times of relaxation, as exhibited by the sludge. When $\eta_{3}$ is much higher than $\eta_{2}$ and $E_{1}$ is the same order of magnitude as $E_{2}$, the dashpot $\eta_{3}$ needs a much longer time to recover itself. So, with respect to a relatively short time scale, the Burgers model can simulate the non-recoverable deformation of viscoelastic property. In this case, the term non-recoverable is relative to the relaxation time. When the relaxation time is long enough, the deformation can be considered as non-recoverable with respect to the time scale of compression test. It can also simulate the linear creep and the relaxation behavior in relatively long time scale.

The viscoelastic behavior of sludge in uniaxial compression can thus be predicted by the Burgers model. The constitutive equations for the Burgers model are written in Equations (8) and (9). Once the stress $\sigma(t)$ is applied on the model, each block of the assembly is deformed accordingly. The stress $\sigma(t)$ in each block of component is identical:

$$
\sigma(t)=E_{1} \varepsilon_{1}(t)=E_{2} \varepsilon_{2}(t)+\eta_{2} \frac{d \varepsilon_{2}(t)}{d t}=\eta_{3} \frac{d \varepsilon_{3}(t)}{d t}
$$

with $\varepsilon_{1}$ the strain of the spring $E_{1}, \varepsilon_{2}$ the strain of the spring $E_{2}$ and the dashpot $\eta_{2}$, and $\varepsilon_{3}$ the strain of the dashpot $\eta_{3}$ at instant $t$.

The total strain of the Burgers model $\varepsilon_{\text {Burgers }}(t)$ is the sum of the strain in each block:

$\varepsilon_{\text {Burgers }}(\mathbf{t})=\varepsilon_{1}(\mathbf{t})+\varepsilon_{2}(\mathbf{t})+\varepsilon_{3}(\mathbf{t})$

\subsubsection{Visco-elasto-plastic behavior modelling}

Once the applied stress reaches threshold, the plastic behavior of sludge is activated. A slider specified with the yield stress $\sigma_{\mathrm{c}}$ is connected in series with the Burgers model to simulate the yielding behavior of plasticity (Fig. 3). As the sludge hardening process exhibits a power law between the plastic stress and the plastic deformation (Liang et al., 2017), the Ludwik's equation (Ludwik, 1909 ) is the simplest model to describe it (Equation (10)).

$\sigma_{\mathbf{p}}(\mathbf{t})=\sigma_{\mathbf{c}}+\mathbf{k}_{\mathbf{L}} \varepsilon_{\mathbf{p}}^{\mathbf{n}_{\mathbf{L}}}(\mathbf{t})$

$\mathrm{k}_{\mathrm{L}}(\mathrm{Pa})$ is the coefficient of material strength and $\mathrm{n}_{\mathrm{L}}(-)$ the hardening coefficient of material. The Ludwik's equation is thus used to be the constitutive law for the slider $\sigma_{\mathrm{c}}$. It is used usually to model the hardening of metals and alloys (Devi et al., 2016; Shiva et al., 2017; Ashrafi et al., 2017).

With addition of the hardening plastic behavior, the constitutive equations are thus updated with the plastic stress $\sigma_{\mathrm{p}}(\mathrm{t})$ and the plastic strain $\varepsilon_{\mathrm{p}}(\mathrm{t})$, as shown in Equations (11), (10) and (12). The stress is identical as previously:

$\sigma(\mathbf{t})=\mathbf{E}_{1} \varepsilon_{1}(\mathbf{t})=\mathbf{E}_{2} \varepsilon_{2}(\mathbf{t})+\eta_{2} \frac{\mathbf{d} \varepsilon_{2}(\mathbf{t})}{\mathbf{d t}}=\eta_{3} \frac{\mathbf{d} \varepsilon_{3}(\mathbf{t})}{\mathbf{d t}}=\sigma_{\mathbf{p}}(\mathbf{t})$

The total stain $\varepsilon(t)$ in visco-elasto-plastic regime is the sum of the Burgers strain and the plastic strain:

$\varepsilon(\mathbf{t})=\varepsilon_{\text {Burgers }}(\mathbf{t})+\varepsilon_{\mathbf{p}}(\mathbf{t})=\varepsilon_{1}(\mathbf{t})+\varepsilon_{2}(\mathbf{t})+\varepsilon_{3}(\mathbf{t})+\varepsilon_{\mathbf{p}}(\mathbf{t})$

The assembly of the 5 analogical mechanic elements with 7 parameters to model the rheological behavior of sludge in uniaxial cyclic compression is therefore titled the Burgers-Ludwik model.
3.2.3. Functioning principle of Burgers-Ludwik model in cyclic compression

To simulate the sludge rheological behavior in 1 cycle of loading-unloading test, the Burgers-Ludwik model functions in 3 steps, as summarized in Table 1:

- In loading phase, when the applied stress is lower than the yield stress, the slider is blocked and only the Burgers model is in action. The sludge deforms as a viscoelastic material.

- With the progress of loading phase, the applied stress reaches the yield stress, the slider is activated and the whole BurgersLudwik model in action. The sludge behaves as a visco-elastoplastic body with hardening effect up to the end of loading phase.

- In unloading phase, the slider is blocked, again, and only the Burgers model is in charge of simulating the viscoelastic recoverable behavior of sludge until the stress reduces to 0 .

\section{Results and discussion}

\subsection{Completion of the optimization procedure of Burgers-Ludwik model}

The Burgers-Ludwik model developed in this work simulates the visco-elasto-plastic behavior of sludge in compression test. The optimization of the proposed visco-elasto-plastic model, namely the determination of the 7 rheological parameters used in the constitutive equations, has to take into account the 3 steps of functioning principle with specific initial and limit conditions. The standard procedure previously described (section 2.5) has thus to be adapted. By considering the limit of the estimated yield stress (Liang et al., 2017), the experimental strain data $\varepsilon_{\exp }$ obtained from loading phase can be classified into viscoelastic strain $\varepsilon_{\exp }$, ve and visco-elasto-plastic strain. The latter is actually the sum of the viscoelastic strain and the plastic strain $\varepsilon_{\text {exp, p }}$, as expressed in Table 2.

For the unloading phase, the measured strain values $\varepsilon_{\text {exp }}$ consist of the maximum plastic strain $\varepsilon_{\text {exp. p.max }}$ obtained at the end of the loading phase and the discharged viscoelastic strain $\varepsilon_{\text {expd, ve }}$ due to sludge recovery (Table 2). So, in order to retrieve the absolute discharged viscoelastic strain data $\varepsilon_{\text {expd, ve, an assistant additional }}$ parameter $\varepsilon_{\mathrm{p}, \max }$ will have to be introduced into the optimization and then matched with $\varepsilon_{\exp \text {, p.max. }}$

\subsubsection{Determination of model parameters in visco-elastic regime}

This assistant parameter $\varepsilon_{\mathrm{p}, \max }$ is assumed to be the maximum value of plastic strain at the end of loading phase (Fig. 5 (a)). It remains constant in the entire unloading phase because it represents the permanent deformation generated by the previous plastic hardening behavior. By subtracting this constant, the unloading strain data consists of pure viscoelastic deformation and thus can be directly used for regressing calculation. The estimation of this parameter is based on the experimental results in cyclic compression tests (Liang et al., 2017): it varies from 0.05 to 0.21 . So its initial value is set to 0.10 .

The residual between experimental results and calculated values is also modified to take into account the implementation of this first stage:

$\left.\left.\operatorname{Residu}=\sum_{\mathbf{i}} \frac{\varepsilon_{\text {cal,ve }}^{\mathbf{i}}-\varepsilon_{\text {exp }}^{\mathbf{i}}}{\varepsilon_{\text {exp }}^{\mathbf{i}}}\right)^{2}+\frac{\varepsilon_{\text {cal,ve }}^{\mathbf{i}}+\varepsilon_{\mathbf{p}, \text { max }}-\varepsilon_{\text {expd,ve }}^{\mathbf{i}}}{\varepsilon_{\text {expd,ve }}^{\mathbf{i}}}\right)^{2}$

It contains two parts: one is the squared difference between 
Table 1

Modelling division of sludge behavior in 1 cycle compression with the Burgers-Ludwik model.

\begin{tabular}{llll}
\hline Phase & Rheological regime & & Model in action \\
\hline Loading & $|\sigma(\mathrm{t})|<\sigma_{\mathrm{c}}$ & Viscoelastic & Burgers \\
& $|\sigma(\mathrm{t})| \geq \sigma_{\mathrm{c}}$ & Visco-elasto-plastic & Burgers-Ludwik \\
Unloading & $|\sigma(\mathrm{t})|<\sigma_{\mathrm{c}}$ & Viscoelastic & Burgers \\
\hline
\end{tabular}

Table 2

Constitution of experimental strain values in 1 cycle compression test.

\begin{tabular}{llll}
\hline Phase & Rheological regime & & \\
\hline Loading & $|\sigma(\mathrm{t})|<\sigma_{\mathrm{c}}$ & Constitution of experimental strain data \\
& $|\sigma(\mathrm{t})| \geq \sigma_{\mathrm{c}}$ & Visco-elastic & $\varepsilon_{\text {exp }}=\varepsilon_{\text {exp }, \text { ve }}$ \\
Unloading & $|\sigma(\mathrm{t})|<\sigma_{\mathrm{c}}$ & Viscoelastic & $\varepsilon_{\text {exp }}=\varepsilon_{\text {exp, ve }}+\varepsilon_{\text {exp, p }}$ \\
\hline
\end{tabular}

experimental and calculated values below the yield stress in loading phase and the other is that in unloading phase which takes into account the preset permanent strain $\left(\varepsilon_{\text {p.max }}\right)$. In this second part, $\varepsilon_{\text {cal }}$ ve consists of the entire viscoelastic strains calculated with all the stress data measured $\left(\sigma_{\exp }\right)$ in unloading phase. Thus, the optimization of the Burgers model can be achieved by using the experimental data of pure viscoelastic behavior in both loading and unloading phases.

As previously described in section 2.5 , the initial values of the five parameters are set to 50, 100 and $150 \%$ of their estimated values. Thus, there are in total $3^{5}=243$ sets of initial values to be evaluated in multiple regression calculations to obtain 243 groups of identified model parameters. The distribution of the identified parameters indicates the uniqueness of attractor in the scope of physical significance.

The basins of attraction are computed with the 243 combinations of preset initial values of parameters for the uniaxial compression cycle at a speed of $0.5 \mathrm{~mm} / \mathrm{s}$ (Liang et al., 2017). Their logarithms are represented because of the different orders of magnitude (Fig. 4 (a)).

Except for the viscosity $\eta_{3}$, all the other 4 parameters and the final residuals of the least squares have very few variations, so they are very similar to horizontal lines in Fig. 4 (a). The parameter $\eta_{3}$ is slightly more sensitive to its initial value, but the optimized results remain in the same order of magnitude. Moreover, the residuals are all in the order of magnitude of $10^{-4}$, so all the optimized values reach the same quality of regression. This also means that small changes in the initial values of the model parameters still lead to the same solution of the differential constitutive equations. It proves the uniqueness of the attractor in the scope of this research. So all the optimized results are kept to calculate the confidence intervals for finer selection. The $\mathrm{Cl}_{\alpha}$ at a $5 \%$ significance level $\alpha$ are presented in Table 3 and illustrated for $\eta_{3}$ on Fig. 4 (b). All the optimized values are consistent with the estimated ones obtained via cyclic, creeping and relaxation compression tests.

Using the definitive values of viscoelastic properties, the sludge response to cyclic compression is simulated with input $\sigma_{\text {exp }}$. The corresponding viscoelastic strain $\varepsilon_{\mathrm{cal}}$, ve is recalculated and then plotted in Fig. 5 (a) for the considered mechanical trial. It can be observed that the calculated values of the strain are closed to the experimental ones in the viscoelastic regime. The deviation between the experimental and simulated results corresponds to the permanent strain $\left(\varepsilon_{\text {exp }, p}\right)$ due to plastic hardening effect (Table 2 ). The difference between the end of loading phase and the beginning of the unloading phase is actually the parameter $\varepsilon_{\text {p.max }}$.

Finally, at this stage, five model parameters $\left(P=\left[E_{1}, E_{2}, \eta_{2}\right.\right.$, $\left.\varepsilon_{\mathrm{p}, \max } \eta_{3}\right]$ ) are determined with the experimental data in viscoelastic regime. Then, the other 3 parameters $\sigma_{\mathrm{c}}, \mathrm{k}_{\mathrm{L}}$ and $\mathrm{n}_{\mathrm{L}}$ of the Ludwik's equation can be determined with the data in sludge hardening regime.

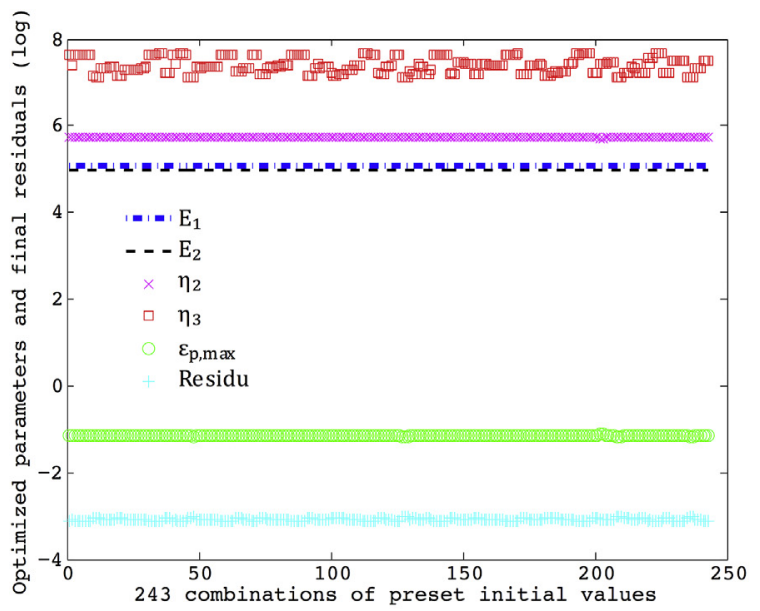

(a)

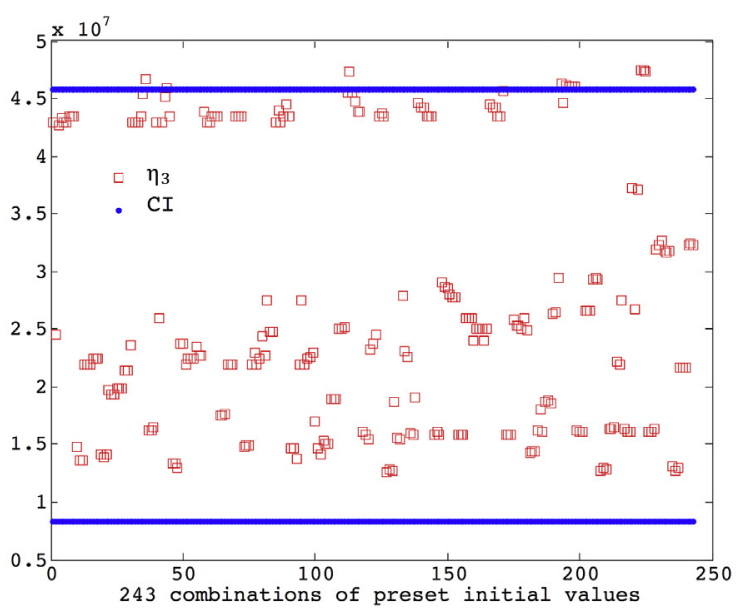

(b)

Fig. 4. (a) Logarithmic values of optimized parameters and residuals of least squares obtained by non linear multiple regression analysis; (b) confidence intervals for optimized values of. $\eta_{3}$ 


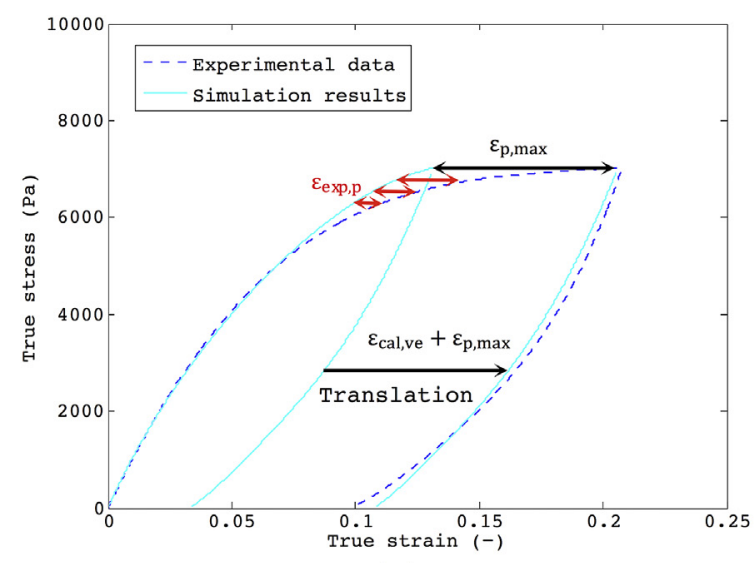

(a)

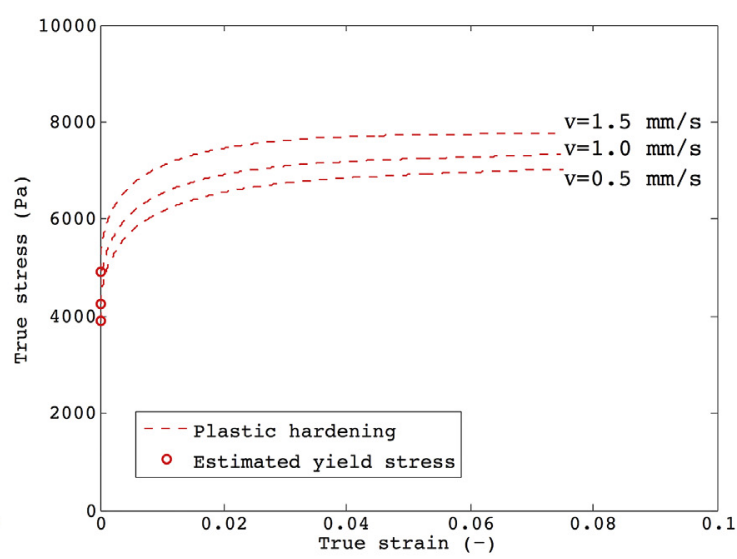

(b)

Fig. 5. (a) Simulation results of Burgers model; (b) pure plastic hardening behavior at increasing compression speed.

Table 3

Minimum and maximum values of optimized parameters and residuals of least squares.

\begin{tabular}{llllll}
\hline & $\mathrm{E}_{1}(\mathrm{~Pa})$ & $\mathrm{E}_{2}(\mathrm{~Pa})$ & $\eta_{2}$ (Pa.s) & $\eta_{3}$ (Pa.s) & Residu (-) \\
\hline Min. & $1.1510^{5}$ & $9.110^{4}$ & $4.810^{5}$ & $1.310^{7}$ & $7.510^{-4}$ \\
Max. & $1.1810^{5}$ & $9.610^{4}$ & $5.410^{5}$ & $4.810^{7}$ & $9.410^{-4}$ \\
\hline
\end{tabular}

\subsubsection{Optimization of Ludwik's equation}

As previously explained, the plastic hardening behaviors ( $\sigma_{\exp }$ in function of $\varepsilon_{\text {exp, p }}$ ) can be plotted for the considered trial (Fig. 5 (b)). On the basis of this behavior, the three hardening parameters in the Ludwik's equation (yield stress $\sigma_{\mathrm{c}}$, index of material strength $\mathrm{k}_{\mathrm{L}}$ and index of plastic hardening $\mathrm{n}_{\mathrm{L}}$ in Equation (10) can be optimized by using the Equation (10) and the least-squares methods and the following residual:

$\left.\operatorname{Residu}=\sum_{\mathbf{i}} \frac{\varepsilon_{\text {cal,p }}^{\mathbf{i}}-\varepsilon_{\text {exp }, \mathbf{p}}^{\mathbf{i}}}{\varepsilon_{\text {exp }, p}^{\mathbf{i}}}\right)^{2}$

The slope of the hardening curves (Fig. 5 (b)) decreases very rapidly at the beginning of the plastic regime $\left(\varepsilon_{\mathrm{p}}<0.02\right)$, but very slowly with the progress of compression. The plastic hardening effect is significant. So the index of material strength $\mathrm{k}_{\mathrm{L}}$ should be in the same order of magnitude as yield stresses, and the index of material hardening $n_{L}$ should be lower than 0.5 , because $n_{L}$ is closer to 0 when plastic effect is stronger. Therefore, $\mathrm{k}_{\mathrm{L}}$ and $\mathrm{n}_{\mathrm{L}}$ are preset at $4000 \mathrm{~Pa}$ and 0.4 as initial values for the model optimization by multiple regression analysis.

Finally, the 7 parameters of the proposed visco-elasto-plastic model, namely the Burgers-Ludwik model, can be optimized with regression analysis by using the described two-step procedure. The numerical simulation of the behavior of sludge in 1 cycle compression test is presented in Fig. 6. The rheological properties determined by the model parameter optimization procedure can reproduce perfectly the sludge behavior.

\subsection{Sensitivity of model to operating parameters}

The definitive values of the Burgers model are presented in Table 4 with respect to the increasing compression speeds $\mathrm{v}$ in cyclic tests (Liang et al., 2017) to identify the impacts on the sludge viscoelastic properties.

All the optimized values are consistent with the estimated ones obtained via cyclic, creeping and relaxation compression tests. The variation with operating conditions of elastic moduli and viscosity $\eta_{3}$ is not clear, but the viscosity $\eta_{2}$ seems to be sensitive to compression speed. Indeed, it decreases more than $60 \%$ when the compression speed increases 3 times what confirms the higher flowability and the shear thinning property (Liang et al., 2017). This effect is not taken into account by the model and it would have been done in a further development. The yield stress increases by $26 \%$ when compression speed is 3 times higher, confirming the growing influence of viscoelasticity effect (Liang et al., 2017). The index of material strength and hardening $\left(\mathrm{k}_{\mathrm{L}}\right.$ and $\left.\mathrm{n}_{\mathrm{L}}\right)$ decrease about $8 \%$ each: increasing compression speed makes the sludge slightly loosened, but it doesn't modify its plastic behavior.

\subsection{Sensitivity of model to structural changes in material}

Fig. 6 presents results for the sludge after a premixing, which is known to modify both the microstructure of sludge (Charlou, 2014) and the moisture distribution (Liang et al., 2016). The model is also able to reveal the effect of the mixing on rheological behavior of sludge. The impact of mixing on sludge rheological properties can be now clearly quantified (Table 5). It is reminded that the result without mixing is different from the previous table because it was carried out another day what means with a different sludge. Mixing doesn't modify the elastic moduli. The viscosity $\eta_{2}$ is less sensible to mixing in comparison with increasing compression speed, and the variation of $\eta_{3}$ is not significant in comparison with the larger confidence interval. The yield stress and the index of material strength decreased about $28 \%$ and $26 \%$, respectively. They are the most sensitive parameters to change of microstructure. Mixing had no significant impact on the index of material hardening, neither.

\section{Conclusion}

The rheological behavior of mechanically dewatered sewage sludges is complex but essential as it affects almost all treatment, utilization and disposal operations. In this work, a specific methodology coupling experiments and modelling is developed to characterize the rheological and textural properties of highly concentrated sewage sludge between the liquid and plastic limits. It is based on a uniaxial compression method presented in a previous paper (Liang et al., 2017). Complementary creep and relaxation mechanical tests are adapted to confirm the viscoelastic property of sludge samples in a longer scale of time. 


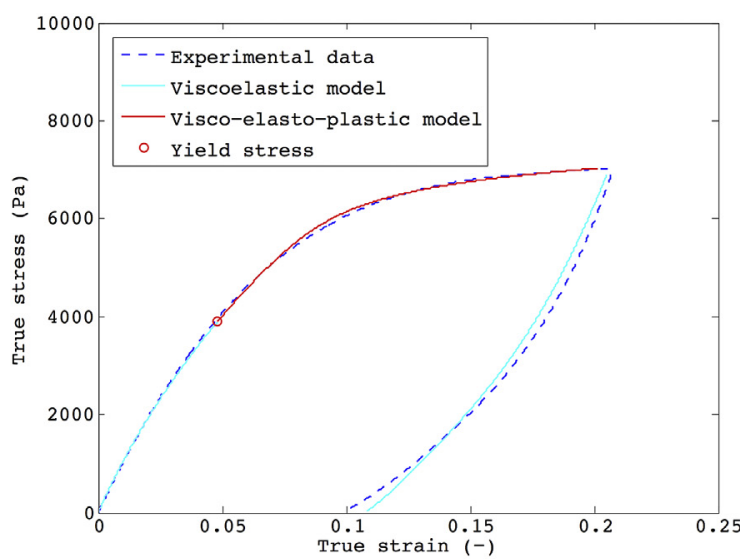

(a)

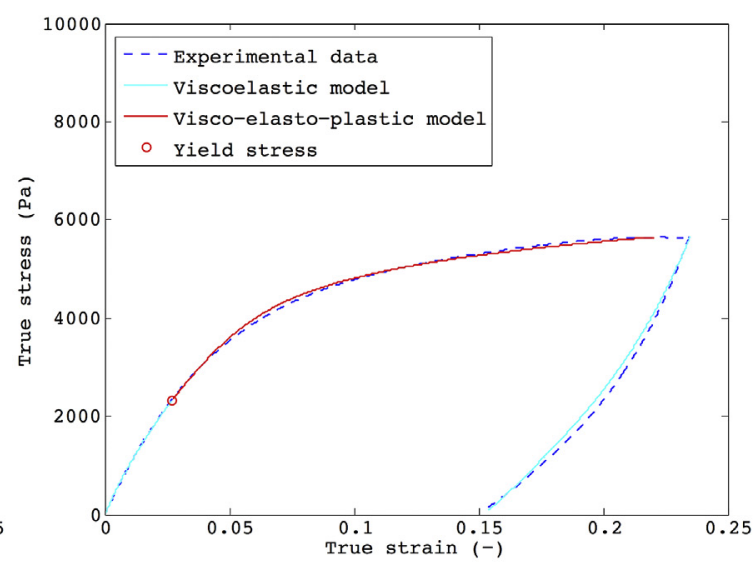

(b)

Fig. 6. Simulation of sludge rheological behavior with Burgers-Ludwik model and determined parameters: (a) original raw sludge sample; (b) premixed sludge sample (20 min of premixing).

Table 4

Definitive values of model parameters for original sludge tested with increasing compression speed.

\begin{tabular}{|c|c|c|c|c|c|c|c|}
\hline \multirow[t]{2}{*}{$\mathrm{v}(\mathrm{mm} / \mathrm{s})$} & \multicolumn{4}{|c|}{ Burgers model } & \multicolumn{3}{|c|}{ Ludwik's equation } \\
\hline & $\mathrm{E}_{1}\left(10^{5} \mathrm{~Pa}\right)$ & $\mathrm{E}_{2}\left(10^{4} \mathrm{~Pa}\right)$ & $\eta_{2}\left(10^{5} \mathrm{~Pa} \mathrm{~s}\right)$ & $\eta_{3}\left(10^{7} \mathrm{Pas}\right)$ & $\sigma_{\mathrm{c}}(\mathrm{Pa})$ & $\mathrm{k}_{\mathrm{L}}(\mathrm{Pa})$ & $\mathrm{n}_{\mathrm{L}}(-)$ \\
\hline 0.5 & 1.16 & 9.23 & 5.36 & 2.24 & 3907 & 4299 & 0.1213 \\
\hline 1.0 & 1.18 & 10.07 & 2.87 & 1.02 & 4261 & 4204 & 0.1159 \\
\hline 1.5 & 1.42 & 9.69 & 1.81 & 0.84 & 4916 & 3954 & 0.1121 \\
\hline
\end{tabular}

Table 5

Definitive values of model parameters for premixed sludge.

\begin{tabular}{|c|c|c|c|c|c|c|c|}
\hline \multirow[t]{2}{*}{ Mixing (min) } & \multicolumn{4}{|c|}{ Burgers model } & \multicolumn{3}{|c|}{ Ludwik's equation } \\
\hline & $\mathrm{E}_{1}\left(10^{5} \mathrm{~Pa}\right)$ & $\mathrm{E}_{2}\left(10^{5} \mathrm{~Pa}\right)$ & $\eta_{2}\left(10^{5} \mathrm{~Pa} \mathrm{~s}\right)$ & $\eta_{2}\left(10^{7}\right.$ Pa s) & $\sigma_{C}(\mathrm{~Pa})$ & $K_{L}(\mathrm{~Pa})$ & $n_{L}(-)$ \\
\hline 0 & 1.23 & 0.94 & 5.82 & 1.56 & 3237 & 6467 & 0.1734 \\
\hline 5 & 1.22 & 0.91 & 5.56 & 2.07 & 3243 & 5686 & 0.1595 \\
\hline 20 & 1.20 & 0.99 & 3.44 & 1.96 & 2331 & 4777 & 0.1682 \\
\hline
\end{tabular}

The identified visco-elasto-plastic is then modelled with a Burgers-Ludwik model, which is combination of 3 analogical mechanical elements: spring, dashpot and slider. It can simulate the viscoelastic behavior of sludge with creep and relaxation under the yield stress, and the visco-elasto-plastic hardening behavior over the yield stress. The optimization of model parameter is based on the calculation of basins of attraction and confidence intervals with reference to the specified initial conditions (estimated from the mechanical tests).

The rheological properties of sludge samples has been then successfully determined at different operating conditions and structural states obtained by means of a sample mixing.

This methodology will be applied to determine the correlations between rheological properties and other factors of impact, such as solid content, temperature or aging. Finally, the rheological model taking into account these impact factors will be able to predict the sludge behavior in complex treatment process.

\section{Appendix}

list of symbols

$\mathrm{CI}_{\alpha} \quad$ confidence interval at the significance level $\alpha$

E elastic modulus $(\mathrm{Pa})$

$\mathrm{F} \quad$ force $(\mathrm{N})$ $\mathrm{h}_{0} \quad$ sludge sample height $(\mathrm{mm})$

$\mathrm{k}_{\mathrm{L}} \quad$ strength coefficient in Ludwik model (Pa)

$\mathrm{n}_{\mathrm{L}} \quad$ hardening coefficient in Ludwik model (-)

$\mathrm{P}_{\mathrm{i}} \quad$ estimated model parameter

$\bar{P} \quad$ average optimized parameter

Residu model residual $(-)$

$\mathrm{R}_{0} \quad$ sludge sample radius ( $\mathrm{mm}$ )

$\mathrm{S}_{\mathrm{P}} \quad$ standard deviation of optimized parameters

$\mathrm{t} \quad$ time (s)

TS total solid content ( wt \%)

$\mathrm{t}_{\alpha / 2}^{\mathrm{n}-\mathrm{m}} \quad$ fractile of order $1-\alpha / 2$ of the Student's t-distribution at

$(n-m)$ degrees of freedom

VS volatil solid content (wt \%)

Greek symbols

$\begin{array}{ll}\varepsilon & \text { (true) strain }(-) \\ \Delta \mathrm{h} & \text { deformation (mm) } \\ \eta & \text { viscosity (Pa.s) } \\ \sigma & \text { (true) stress (Pa) } \\ \tau & \text { characteristic time (s) }\end{array}$

Indexes

$1 \quad$ spring of the Maxwell model

2 spring and dashpot of the Kelvin Voigt model

3 dashpot of the Kelvin-Voigt model 


$\begin{array}{ll}\text { Burgers } & \text { Burgers model } \\ \text { C } & \text { slider yield (Ludwik model) } \\ \text { cal } & \text { calculated } \\ \text { exp } & \text { experimental } \\ \text { expd } & \text { experimental unloading } \\ \text { f } & \text { creeping } \\ \text { M } & \text { Maxwell model } \\ \text { max } & \text { maximal } \\ \text { p } & \text { plastic } \\ \text { r } & \text { relaxation } \\ \text { ve } & \text { viscoelastic }\end{array}$

\section{References}

Ashrafi, H., Shamanian, M., Emadi, R., Saeidi, N., 2017. A novel and simple technique for development of dual phase steels with excellent ductility. Mater. Sci. Eng. 680, 197-202.

Barnes, H.A., Hutton, J.F., Walters, K., 1998. An Introduction to Rheology. Elsevier Science.

Baudez, J.C., Coussot, P., Thirion, F., 1998. Rheology of sludge of Wastewater Treatment Plant: Preliminary studies for mastering of storage and spreading. (in French, Rhéologie Des Boues de Stations D'épuration: études Préliminaires Pour La Maîtrise Des Stockages et épandages.) Ingénieries - EAT - N 15.

Charlou, C., 2014. Characterization and modelling of sewage sludge flow in paddle dryer (in French, Caractérisation et modélisation de l'écoulement des boues résiduaires dans un sécheur à palettes.). PhD. Thesis. Université de Toulouse, Albi.

Chhabra, R.P., Richardson, J.F., 2011. Non-newtonian Flow and Applied Rheology: Engineering Applications. Butterworth-Heinemann.

Costa, I., Banos, J., 2015. Tensile creep of a structural epoxy adhesive: experimental and analytical characterization. Int. J. Adhesion Adhes. 59, 115-124.

Coussot, P., 2014. Yield stress fluid flows: a review of experimental data. J. NonNewtonian Fluid Mech. 211, 31-49. September.

Coussot, P., 1997. Mudflow Rheology and Dynamics. Taylor \& Francis.

Devi, R.S.R., Poshal, G., Prasad, K.E., Goud, R.R., 2016. Strain hardening behaviour of hot rolled annealed nickel free nitrogen based austenitic stainess steel. Mater. Today: Proc. 4 (2), 917-926.

Ercan, N., Durmus, A., Kasgoz, A., 2017. Comparing of melt blending and solution mixing methods on the physical properties of thermoplastic polyurethane/ organoclay nanocomposite films. J. Thermoplast. Compos. Mater. 30, 950-970.

Eshtiaghi, N., Markis, F., Dong Yap, S., Baudez, J.C., Slatter, P., 2013. Rheological characterisation of municipal sludge: a review. Water Res. 47 (15), 5493-5510.

Eurostat, Sewage sludge production and disposal from urban wastewater, https:// ec.europa.eu/eurostat/web/products-datasets/-/ten00030, consulted on 26 September 2018

Jiang, J., Wu, J., Poncin, S., Li, H., 2014. Rheological characteristics of highly concentrated anaerobic digested sludge. Biochem. Eng. J. 86, 57-61.

Jiang, Y.Z., Wang, R.H., Zhu, J.B., 2015. Rheological mechanical properties of altered- rock. Mater. Res. Innovat. 19, 349-355.

Liang, F., Sauceau, M., Dusserre, G., Arlabosse, P., 2017. A uniaxial cyclic compression method for characterizing the rheological and textural behaviors of mechanically dewatered sewage sludge. Water Res. 113, 171-180.

Liang, F., Chen, X., Mao, H., Sauceau, M., Arlabosse, P., Wang, F., Chi, Y., 2016. The microstructure: a critical factor in characterising the sticky properties of highly concentrated sewage sludge. In: 6th International Conference on Engineering for Waste and Biomass Valorisation.

Lin, Y.M., Sharma, P.K., van Loosdrecht, M.C.M., 2013. The chemical and mechanical differences between alginate-like exopolysaccharides isolated from aerobic flocculent sludge and aerobic granular sludge. Water Res. 47 (1), 57-65.

Lotito, V., Lotito, A.M., 2014. Rheological measurements on different types of sewage sludge for pumping design. J. Environ. Manag. 137, 189-196.

Ludwik, P., 1909. Elements of Technical Mechanics. Springer, Berlin.

Malkin, A.Y., Isayev, A.I., 2006. Rheology Concepts, Methods \& Application. ChemTec Publishing.

Meerts, M., Cardinaels, R., Oosterlinck, F., Courtin, C.M., Moldenaers, P., 2017. The interplay between the main flour constituents in the rheological behaviour of wheat flour dough. Food Bioprocess Technol. 10, 249-265.

Moreira, R., Chenlo, F., Arufe, S., Rubinos, S.N., 2015. Physicochemical characterization of white, yellow and purple maize flours and rheological characterization of their doughs. J. Food Sci. Technol. 52, 7954-7963.

Mori, M., Isaac, J., Seyssiecq, I., Roche, N., 2008. Effect of measuring geometries and of exocellular polymeric substances on the rheological behaviour of sewage sludge. Chem. Eng. Res. Design 86 (6), 554-559, 11th Congress of the French Chemical Engineering Society.

Mori, M., Seyssiecq, I., Roche, N., 2006. Rheological measurements of sewage sludge for various solids concentrations and geometry. Process Biochem. 41 (7), $1656-1662$.

Morrison, F.A., 2001. Understanding Rheology of Dispersions. Oxford University Press.

Ratkovich, N., Horn, W., Helmus, F.P., Rosenberger, S., Naessens, W., Nopens, I., Bentzen, T.R., 2013. Activated sludge rheology: a critical review on data collection and modelling. Water Res. 47 (2), 463-482.

Seyssiecq, I., Ferrasse, J.H., Roche, N., 2003. State-of-the-Art: rheological characterisation of wastewater treatment sludge. Biochem. Eng. J. 16 (1), 41-56.

Shiva, V., Goyal, S., Sandhya, R., Laha, K., Bhaduri, A.K., 2017. Flow behaviour of modified 9Cr-1Mo steel at elevated temperatures. Trans. Indian Inst. Met. 70 (3), 589-596.

Tadros, T, 2010. Rheology of Dispersions, first ed. WILEY-VCH Verlag GmbH \& Co.

Tanner, R.I., Walters, K., 1998. Rheology: an Historical of Perspective. Elsevier Science.

Yu, J., Santos, P.H.S., Campanella, O.H., 2012. A study to characterize the mechanical behavior of semisolid viscoelastic systems under compression chewing - case study of agar gel. J. Texture Stud. 43 (6), 459-467.

Zhang, C., Cao, P., Pu, C., Liu, J., Wen, P., 2014. Integrated identification method of rheological model of sandstone in sanmenxia bauxite. Trans. Nonferrous Metals Soc. China 24 (6), 1859-1865.

Zhang, J., Xue, Y., Eshtiaghi, N., Dai, X., Tao, W., Li, Z., 2017. Evaluation of thermal hydrolysis efficiency of mechanically dewatered sewage sludge via rheological measurement. Water Res. 116, 34-43. 